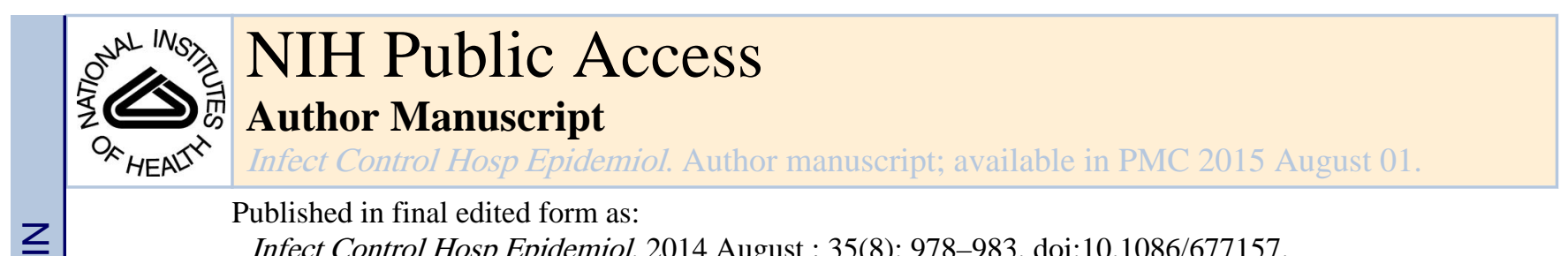

Infect Control Hosp Epidemiol. 2014 August ; 35(8): 978-983. doi:10.1086/677157.

\title{
Rising Rates of Carbapenem-Resistant Enterobacteriaceae in Community Hospitals: A Mixed-Methods Review of Epidemiology and Microbiology Practices in a Network of Community Hospitals in the Southeastern United States
}

\author{
Joshua T. Thaden, MD, PhD' ${ }^{1}$, Sarah S. Lewis, MD $^{1,2}$, Kevin C. Hazen, PhD $^{3}$, Kirk Huslage, \\ BSN, MSPH ${ }^{4}$, Vance G. Fowler Jr, MD, MHS ${ }^{1}$, Rebekah W. Moehring, MD, MPH ${ }^{1,2,5}$, Luke F. \\ Chen, MBBS, MPH ${ }^{1,2}$, Constance D. Jones, RN, $\mathrm{CIC}^{6}$, Zack S. Moore, MD, MPH ${ }^{6}$, Daniel J. \\ Sexton, $\mathbf{M D}^{1,2}$, and Deverick J. Anderson, MD, MPH ${ }^{1,2}$ \\ ${ }^{1}$ Duke University Division of Infectious Diseases, Durham, North Carolina \\ 2Duke Infection Control Outreach Network, Durham, North Carolina \\ ${ }^{3}$ Duke Clinical Microbiology Laboratory, Duke University, Durham, North Carolina \\ ${ }^{4}$ Statewide Program for Infection Control and Epidemiology, University of North Carolina at \\ Chapel Hill, Chapel Hill, North Carolina \\ ${ }^{5}$ Durham Veterans Affairs Medical Center, Durham, North Carolina \\ ${ }^{6}$ North Carolina Division of Public Health, Raleigh, North Carolina
}

\begin{abstract}
OBJECTIVE-Describe the epidemiology of carbapenem-resistant Enterobacteriaceae (CRE) and examine the effect of lower carbapenem breakpoints on CRE detection.

DESIGN-Retrospective cohort.

SETTING-Inpatient care at community hospitals.

PATIENTS-All patients with CRE-positive cultures were included.

METHODS-CRE isolated from 25 community hospitals were prospectively entered into a centralized database from January 2008 through December 2012. Microbiology laboratory practices were assessed using questionnaires.
\end{abstract}

RESULTS-A total of 305 CRE isolates were detected at 16 hospitals (64\%). Patients with CRE had symptomatic infection in 180 cases (59\%) and asymptomatic colonization in the remainder (125 cases; 41\%). Klebsiella pneumoniae (277 isolates; $91 \%$ ) was the most prevalent species. The majority of cases were healthcare associated (288 cases; 94\%). The rate of CRE detection

(C) 2014 by The Society for Healthcare Epidemiology of America. All rights reserved.

Address correspondence to Joshua T. Thaden, MD, PhD, Clinical Fellow in Division of Infectious Diseases, Duke University Medical Center, 315 Trent Drive, Durham, NC 27710 (joshua.thaden@duke.edu).

Potential conflicts of interest. All authors report no conflicts of interest relevant to this article. All authors submitted the ICMJE Form for Disclosure of Potential Conflicts of Interest, and the conflicts that the editors consider relevant to this article are disclosed here. 
increased more than fivefold from 2008 ( 0.26 cases per 100,000 patient-days) to 2012 ( 1.4 cases per 100,000 patient-days; incidence rate ratio (IRR), 5.3 [95\% confidence interval (CI), 1.2222.7]; $P=.01$ ). Only 5 hospitals (20\%) had adopted the 2010 Clinical and Laboratory Standards Institute (CLSI) carbapenem breakpoints. The 5 hospitals that adopted the lower carbapenem breakpoints were more likely to detect CRE after implementation of breakpoints than before (4.1 vs 0.5 cases per 100,000 patient-days; $P<.001$; IRR, 8.1 [95\% CI, 2.7-24.6]). Hospitals that implemented the lower carbapenem breakpoints were more likely to detect CRE than were hospitals that did not (3.3 vs 1.1 cases per 100,000 patientdays; $P=.01$ ).

CONCLUSIONS-The rate of CRE detection increased fivefold in community hospitals in the southeastern United States from 2008 to 2012. Despite this, our estimates are likely underestimates of the true rate of CRE detection, given the low adoption of the carbapenem breakpoints recommended in the 2010 CLSI guidelines.

Carbapenem-resistant Enterobacteriaceae (CRE) have emerged as a global threat. ${ }^{1}$ Patients with CRE infections have adverse outcomes, including mortality risk ranging from $48 \%$ $71 \% .^{2-5}$ Multidrug-resistant organisms, such as CRE, have been labeled as "a serious threat to public health" by the Centers for Disease Control and Prevention (CDC $)^{6(\mathrm{p} 36)}$ and "one of the three greatest threats to human health," by the World Health Organization. ${ }^{7(p 1939)}$ Although CRE are still relatively uncommon in the United States, the rate of carbapenem resistance among Enterobacteriaceae is increasing. Among cases due to Klebsiella pneumoniae, for example, the percentage due to meropenem-resistant strains increased from $0.6 \%$ to $5.4 \%$ from 2004 to 2008 . $^{8}$

Little is known about the epidemiology of CRE in community hospitals, despite the fact that the majority of healthcare in the United States is provided in this setting. ${ }^{9}$ These hospitals often have difficulty identifying trends, given the sporadic occurrence of cases, and as a result are less likely to view CRE as an important pathogen in their facilities.

Despite the global emergence of CRE, no clear consensus has emerged in regard to the method of detection. Because of early studies that showed that some CRE had carbapenem minimum inhibitory concentrations (MICs) in the susceptible range, ${ }^{10}$ the Clinical and Laboratory Standards Institute (CLSI) recently lowered breakpoints for carbapenem antibiotics. ${ }^{11}$ Adherence to the new CLSI breakpoints requires that laboratories take additional steps to validate the lower breakpoints. Many laboratories have not yet adopted the new carbapenem breakpoints, potentially resulting in decreased detection and underestimation of CRE prevalence. The objectives of our study were to (1) describe the epidemiology of CRE in a network of community hospitals in the southeastern United States, (2) evaluate the methods that these hospitals use to detect CRE, and (3) better understand how differences in laboratory methods influence CRE detection.

\section{METHODS}

\section{Participating Hospitals}

This mixed-methods study included data from 25 community hospitals in North Carolina, South Carolina, Virginia, and Georgia from January 2008 through December 2012. All hospitals were members of the Duke Infection Control Outreach Network (DICON), which 
has been described elsewhere. ${ }^{12}$ All 25 hospitals responded to the questionnaire and had complete surveillance data for the entire 5-year period.

\section{Surveillance Data}

Local infection preventionists (IPs) performed prospective surveillance for CRE using standardized protocols in all hospitals. The IPs collected and entered data on patients with CRE isolates into a centralized database, including the following variables: infection versus colonization, year of birth, sex, ethnicity, date of hospital admission, previous admissions to the same hospital during the preceding year, specimen collection data and type, dialysis dependence, and whether admission was from home or another healthcare facility. IPs also entered monthly patient-days for each hospital. Incidence rates were calculated as the number of patients with CRE per 100,000 patient-days. To determine infection versus colonization, IPs examined the medical record and spoke to the primary healthcare providers to evaluate for signs or symptoms consistent with infection. All IPs received identical training and followed identical standard protocols in this regard.

\section{Study Patients and Definitions}

All CRE-positive cultures from the surveillance database were reviewed for inclusion. If an individual patient had multiple hospitalizations during which CRE was detected, only the first isolate and admission were included; thus, a single patient was counted once even if they produced multiple cultures with CRE over the 5-year period. Location of acquisition was defined as follows: (1) "community onset, healthcare associated" was defined as infection or colonization occurring less than 48 hours after admission plus the presence of 1 or more of the following healthcare risk factors: previous hospitalization, surgery, dialysis, or residence in a long-term care facility in the 12 months preceding identification or the presence of an invasive device; (2) "community acquired" was defined as identification occurring less than 48 hours after admission without 1 of the above healthcare risk factors; and (3) "hospital onset" was defined as identification that occurred 48 hours or more after hospital admission. ${ }^{13}$ CRE were defined using CDC definitions based on phenotypic susceptibility. ${ }^{6}$ Enterobacteriaceae had to be nonsusceptible to 1 or more of the carbapenem antibiotics according to the breakpoints of the individual microbiology laboratories. CRE phenotype was determined by automated platforms according to local practice (Vitek 2 [bioMérieux] or Microscan Walk-away [Dade Behring]) using panels provided by the manufacturer for this purpose.

\section{Questionnaire Data}

Qualitative laboratory data were obtained from all 25 hospitals using questionnaires. First, the North Carolina Department of Public Health (NC DPH) sent surveys to all North Carolina acute care hospital microbiology laboratories and hospital IPs in July 2012. The survey requested information regarding testing methods used to identify CRE, the automated MIC system in use, whether the 2010 CLSI carbapenem breakpoints had been implemented, and what additional actions were taken if a CRE was detected. Thirteen study hospitals participated in this survey. 
Hospitals from which no survey data were available, including those in North Carolina that did not respond to the survey and all DICON-affiliated hospitals in South Carolina, Virginia, and Georgia, received a telephone call to the hospital microbiology laboratory supervisor from April to September 2013. Telephone survey questions mirrored the questionnaire used by the NC DPH. In addition, the telephone survey addressed local site practices of active CRE surveillance. Data from 12 additional hospitals were obtained through this second round of telephone surveys.

\section{Statistics}

Descriptive statistics were used to characterize the epidemiology of CRE in the 25 study hospitals with complete surveillance data. Poisson regression was used to determine trends in the rates of CRE detection and to calculate relative rates and 95\% confidence intervals (CIs). Overdispersion was observed and corrected using the Pearson $\chi^{2}$ as the dispersion parameter. Generalized estimating equation regression using a Poisson distribution was used to test for trend while controlling for nonrandom clustering of each outcome. Denominator data were normalized by $\log$ transformation. SAS software version 9.3 was used for all calculations (SAS Institute). A 2-sided $P$ value of less than or equal to .05 was considered significant for all tests.

One of the 25 hospitals was known to have endemic CRE and was excluded from trend analyses (over $50 \%$ of the detected CRE strains in the network came from this hospital). For the analysis involving rates of CRE detection based on adoption of the 2010 CLSI guidelines, we performed 2 comparisons using Poisson regression methods described above. First, among the microbiology laboratories that adopted the 2010 guidelines $(n=5)$, we compared rates of CRE detection before and after the guidelines were adopted and implemented. Second, we limited the time period to 2011-2012 and compared rates at switch hospitals to those at nonswitch hospitals.

\section{RESULTS}

\section{Surveillance Data}

Bed size for the 25 study hospitals ranged from 100 to 657 (median, 210 beds). A total of 305 unique patients with CRE from 16 hospitals were identified during 7,312,847 patientdays of surveillance (cumulative incidence rate of CRE detection, 4.17 cases per 100,000 patient-days).

Patients with CRE had symptomatic infection in 180 cases (59\%) and asymptomatic colonization in the remainder (125 cases; $41 \%$; Table 1$)$. K. pneumoniae $(n=277 ; 91 \%)$ was the most prevalent species. The most common anatomical sites involved were urine $(n=$ $181 ; 59 \%)$, sputum $(n=51 ; 17 \%)$, and blood $(n=21 ; 7 \%)$. The majority of cases were healthcare associated; $184(60 \%)$ were community-onset, healthcare-associated cases, and 104 (34\%) were hospital-onset, healthcare-associated cases. Of the 184 community-onset, healthcare-associated CRE cases, $103(56 \%)$ were in patients who were admitted from a nursing home or other extended care facility. 


\section{Laboratory Questionnaire Data}

Questionnaire data from the clinical microbiology laboratories of 25 community hospitals are presented in Table 2. Twenty hospitals (80\%) had not adopted the 2010 CLSI breakpoints for carbapenems. All laboratories used automated MIC detection systems; 13 (52\%) of the hospitals used the Vitek 2 system, and 12 (48\%) used the Microscan system. Verification of carbapenemase production was performed in house with the modified Hodge test in 10 hospitals (40\%), by forwarding on to an outside laboratory for testing in 13 hospitals (52\%), or not at all in 2 hospitals (6\%). Twenty-three laboratories (92\%) reported that, if CRE were detected, they would notify the local infection control personnel. No hospitals had adopted active CRE surveillance programs.

\section{Trends in CRE Detection}

Trends and rate analyses were limited to the 25 hospitals with complete surveillance and questionnaire data. When data from 1 additional hospital where CRE was known to be endemic were excluded, the incidence rate of CRE detection was 1.2 cases per 100,000 patient-days and the median rate of CRE detection per hospital was 0.79 cases per 100,000 patient-days (interquartile range [IQR], 0-1.71). The rate of CRE detection increased more than fivefold from 2008 ( 0.26 cases per 100,000 patient-days) to 2012 (1.4 cases per 100,000 patient-days; incidence rate ratio [IRR], 5.3 [95\% CI, 1.22- 22.7]). This increase remained statistically significant even after adjusting for clustering of CRE by hospital $(P=$. 01; Figure 1).

The rate of CRE detection was significantly higher among hospitals that used the Vitek 2 system as opposed to the Microscan (2.47 vs 0.54 CRE isolates detected per 100,000 patient-days; $P=.01$; Table 3 ). Hospitals that verified the presence of carbapenemases with an in-house modified Hodge test tended to detect CRE at a higher rate than those that sent the strains to a referral laboratory (2.36 vs 1.00 CRE isolates detected per 100,000 patientdays; $P=.07)$. Finally, hospitals that would notify infection control when CRE were detected tended to detect more CRE than those hospitals that would not (mean, 1.64 vs 0 CRE isolates detected per 100,000 patient-days; $P=.16$ ).

The 5 hospitals that adopted the new CLSI carbapenem breakpoints were more likely to detect CRE after implementation of the breakpoints than before implementation (4.1 vs 0.5 isolates per 100,000 patient-days; IRR, 8.1 [95\% CI, 2.7- 24.6]; $P<.001$ ). Finally, hospitals that implemented the CLSI carbapenem breakpoints were more likely to detect CRE than were hospitals that did not (3.6 vs 1.1 isolates per 100,000 patient-days; $P=.01$ ).

In light of the above findings, we performed an exploratory analysis using the Poisson model constructed above. After controlling for whether the new CLSI carbapenem breakpoints had been adopted and implemented, detection of CRE still increased during the time period, although the trend was no longer statistically significant $(P=.08)$. A separate Poisson regression model limited to the 19 hospitals that had not yet adopted the guidelines also demonstrated an increasing trend in CRE detection $(P=.02)$. These findings suggest that the increasing CRE trend is related to both changes in testing and increasing endemicity. 


\section{DISCUSSION}

Our study is, to our knowledge, the first to use mixed methods to describe and compare epidemiologic trends and microbiologic practices in a large number of community hospitals. There are major and interrelated conclusions from our analysis: (1) the rate of CRE detection in community hospitals is rapidly and significantly increasing, (2) microbiology laboratories in community hospitals use widely varying techniques for detection and reporting of CRE, and (3) more preparation is needed to combat this emerging threat.

The rate of CRE detection increased more than fivefold in our network of community hospitals from 2008 to 2012. The higher rate of CRE infection likely stems from a combination of factors, including increased use of broad-spectrum carbapenems, the ease with which carbapenemase enzymes can be transmitted among bacteria, and increased transmission between patients in healthcare settings. For example, $94 \%$ of cases of CRE infection detected in our study were healthcare associated. Given the recent finding that $30 \%$ of patients in long-term acute care hospitals are colonized with carbapenemase- producing K. pneumoniae, ${ }^{14}$ there are likely to be reservoirs of CRE within acute care hospitals, skilled nursing facilities, and hemodialysis centers that are driving the increase in CRE transmission in healthcare settings.

The use of more sensitive detection methods by clinical microbiology laboratories also contributes to recent increases in CRE. Microbiology laboratories in our network used different automated MIC systems, carbapenem breakpoints, and methods to verify the presence of carbapenemases (if any verification was done at all). Rates of CRE detection varied on the basis of these different approaches. We believe the low adoption of the decreased carbapenem breakpoints from the 2010 CLSI guidelines results in a significant underestimation in the true rate of CRE detection in our network of community hospitals. In our cohort, the rate of CRE detection was over threefold higher in hospitals that adopted and implemented the 2010 CLSI guidelines. Interestingly, there was a statistically significant association between the use of the Vitek 2 automated MIC system and increased rate of CRE detection. Previous head-to-head studies have demonstrated that the Microscan is at least equivalent to if not superior to the Vitek 2 system in detecting CRE. ${ }^{15}$ Thus, the cause of this association is unclear. It is conceivable that hospitals using Vitek 2 in this study had other confounding factors that caused this observation (eg, higher colonization pressure or worse CRE prevention practices).

The relatively low rate of CRE detection among the community hospitals in this study punctuates the importance of using networks of hospitals to identify larger epidemiologic trends and to better understand the importance of microbiology laboratory practices in detecting these pathogens. The overall number of CRE detected in individual hospitals is generally not sufficient to make firm conclusions regarding trends. As a result, practitioners in these single institutions would find it difficult to detect the increased rate of CRE detection or assess how variability in laboratory practices influences CRE detection. Analysis of surveillance data from the DICON network of community hospitals, however, has demonstrated that multiple epidemiologically important bacteria — extended-spectrum 
$\beta$-lactamase-producing bacteria ${ }^{13}$ and now CRE-are present and increasing in number in community hospitals in the United States.

There are several limitations in this study. First, the CRE surveillance data were recorded by local hospital IPs after interpretation of microbiology data. There is inherent subjectivity in surveillance practices despite the use of standard protocols and database. Given the overall low number of CRE present in community hospitals, this variability could be significant and skew the data in unclear ways. We recently performed a retrospective analysis, however, that confirmed that the definition used for "multidrug-resistant" gram-negative phenotypes by IPs detected $100 \%$ of CRE that occurred at a single tertiary care medical center. ${ }^{16}$ This issue is the reality of infection surveillance in community hospitals and further highlights the nationwide challenge in detecting and controlling this emerging threat. Second, this study used qualitative data from microbiology laboratory directors regarding laboratory practices, which may be subject to recall bias. We used multiple surveys to gain a more complete data set. Third, we did not collect data on the presence of carbapenemases, and so we are unable to comment on the molecular epidemiology of genes important in carbapenem resistance. Despite these limitations, we believe that this study provides a practical description of the limited data available on incidence of CRE in a multicenter sample of community hospitals and highlights deficiencies in preparedness for this public health threat.

We believe community hospitals in our network have much more work to do to prepare for and respond to CRE. Specifically, 2 focus areas must be developed to prevent CRE transmission: infection control and improved laboratory detection. Hospitals must be vigilant to limit person-to-person transmission. The CDC has outlined basic strategies to decrease transmission, including hand hygiene, contact precautions, healthcare personnel education, limitation of medical device use, patient and staff cohorting, laboratory notification strategies, antimicrobial stewardship, and CRE active screening. ${ }^{6}$ Of note, hospitals in our network have had significant difficulty implementing these recommendations because of resource limitations. No community hospitals in our cohort have yet adopted active surveillance programs for CRE. Finally, microbiology laboratories in these hospitals must make every effort to switch to the new carbapenem breakpoints. For example, all of the hospitals that did not detect a single CRE also did not adopt the new breakpoints. Thus, we remain skeptical that CRE are indeed absent from these hospitals. The inability to detect and control CRE makes in-hospital transmission more likely and could further drive the increasing trend and lead to hospital outbreaks.

In conclusion, our data indicate that the rates of CRE, while still infrequent, are increasing dramatically in community hospitals, where the majority of Americans receive their healthcare. We believe this increase is attributable to growing reservoirs and transmission of CRE and improvement in detection. Overall, we believe the estimates from study hospitals are underestimates of the true incidence in these hospitals. This point underscores the fact that these organisms are increasingly important and relevant in all areas of healthcare, including small community hospitals. Greater adherence to the 2010 CLSI guidelines is necessary to better understand the true prevalence of CRE and to better define what public health measures must be undertaken to prevent further spread of this serious, emerging threat. 


\section{Acknowledgments}

Financial support. D.J.A. was supported by K23-AI095357. V.G.F. was supported by K24-AI093969 and UM1AI0468.

\section{REFERENCES}

1. Nordmann P, Naas T, Poirel L. Global spread of carbapenemase-producing Enterobacteriaceae. Emerg Infect Dis. 2011; 17(10):1791-1798. [PubMed: 22000347]

2. Ben-David D, Kordevani R, Keller N, et al. Outcome of carbapenem resistant Klebsiella pneumoniae bloodstream infections. Clin Microbiol Infect. 2012; 18(1):54-60. [PubMed: 21722257]

3. Borer A, Saidel-Odes L, Riesenberg K, et al. Attributable mortality rate for carbapenem-resistant Klebsiella pneumoniae bacteremia. Infect Control Hosp Epidemiol. 2009; 30(10):972-976. [PubMed: 19712030]

4. Daikos GL, Petrikkos P, Psichogiou M, et al. Prospective observational study of the impact of VIM-1 metallo-beta-lactamase on the outcome of patients with Klebsiella pneumoniae blood-stream infections. Antimicrob Agents Chemother. 2009; 53(5):1868-1873. [PubMed: 19223638]

5. Patel G, Huprikar S, Factor SH, Jenkins SG, Calfee DP. Outcomes of carbapenem-resistant Klebsiella pneumoniae infection and the impact of antimicrobial and adjunctive therapies. Infect Control Hosp Epidemiol. 2008; 29(12):1099-1106. [PubMed: 18973455]

6. Centers for Disease Control and Prevention. [Accessed November 2, 2013] 2012 CRE toolkit: guidance for control of carbapenem-resistant Enterobacteriaceae. http://www.cdc.gov/hai/ organisms/cre/cre-toolkit/index.html. Published 2012.

7. Wise R. The urgent need for new antibacterial agents. J Antimicrob Chemother. 2011; 66(9):19391940. [PubMed: 21700627]

8. Gupta N, Limbago BM, Patel JB, Kallen AJ. Carbapenem-resistant Enterobacteriaceae: epidemiology and prevention. Clin Infect Dis. 2011; 53(1):60-67. [PubMed: 21653305]

9. Agency for Healthcare Research and Quality. [Published 2013. Accessed October 13, 2013] Healthcare Cost and Utilization Project (HCUP).. http://www.ahrq.gov/research /data/hcup/ index.html.

10. Anderson KF, Lonsway DR, Rasheed JK, et al. Evaluation of methods to identify the Klebsiella pneumoniae carbapenemase in Enterobacteriaceae. J Clin Microbiol. 2007; 45(8):2723-2725. [PubMed: 17581941]

11. Clinical and Laboratory Standards Institute (CLSI). Performance Standards for Antimicrobial Susceptibility Testing: Twentieth Informational Supplement. Wayne, PA: CLSI; 2010. CLSI document M100-S20.

12. Anderson DJ, Miller BA, Chen LF, et al. The network approach for prevention of healthcareassociated infections: long-term effect of participation in the Duke Infection Control Outreach Network. Infect Control Hosp Epidemiol. 2011; 32(4):315-322. [PubMed: 21460482]

13. Freeman JT, Sexton DJ, Anderson DJ. Emergence of extendedspectrum $\beta$-lactamase-producing Escherichia coli in community hospitals throughout North Carolina: a harbinger of a wider problem in the United States? Clin Infect Dis. 2009; 49(2):e30-e32. [PubMed: 19522654]

14. Lin MY, Lyles-Banks RD, Lolans K, et al. The importance of long-term acute care hospitals in the regional epidemiology of Klebsiella pneumoniae carbapenemase-producing Enterobacteriaceae. Clin Infect Dis. 2013; 57(9):1246-1252. [PubMed: 23946222]

15. Woodford N, Eastaway AT, Ford M, et al. Comparison of BD Phoenix, Vitek 2, and MicroScan automated systems for detection and inference of mechanisms responsible for carbapenem resistance in Enterobacteriaceae. J Clin Microbiol. 2010; 48(8):2999-3002. [PubMed: 20534805]

16. Lewis, SS.; Moehring, RW.; Chen, LF.; Sexton, DJ.; Anderson, DJ. Program and abstracts of IDWeek. San Francisco, CA: IDWeek; 2013. Defining multidrug resistance (MDR) for gramnegative (GN) infections: who gets the gown?. 2013. Abstract 40634. 


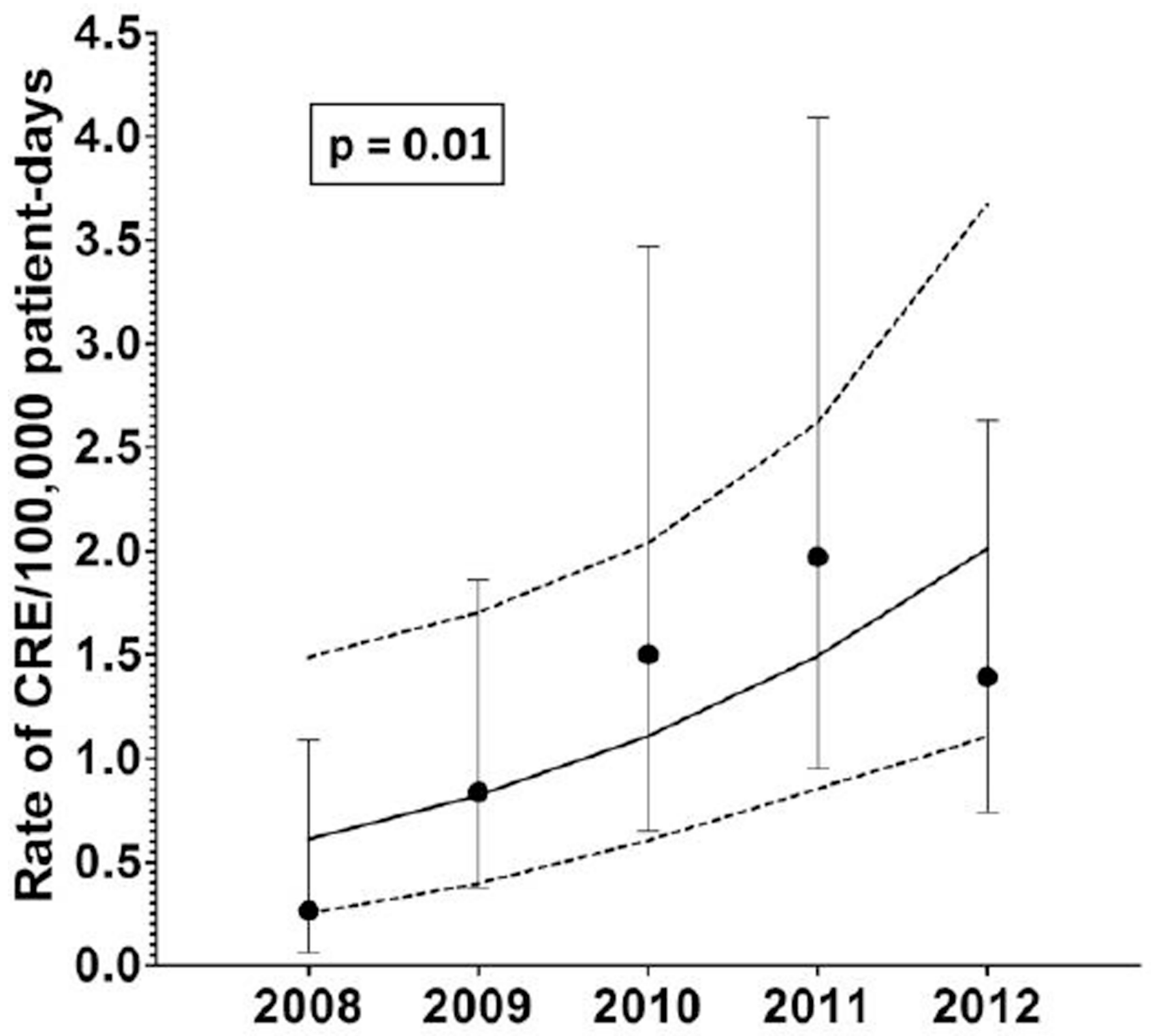

Figure 1.

Trend analysis for carbapenem-resistant Enterobacteriaceae (CRE) detection per 100,000 patient-days from the Duke Infection Control Outreach Network. Mean rates of CRE detection per 100,000 patient-days per year are indicated by black dots, and $95 \%$ confidence intervals (CIs) are indicated with bars. Trend line (solid line) and 95\% CIs for the trend line (dashed lines) were constructed using Poisson regression while controlling for clustering by hospital. The increase in CRE detection over this period is statistically significant $(P=.01)$. 


\section{TABLE 1}

Epidemiology of Carbapenem-Resistant Enterobacteriaceae (CRE) Surveillance Data from the Duke Infection Control Outreach Network, 2008-2012

\begin{tabular}{|c|c|}
\hline Variable & $\begin{array}{c}\text { No. }(\%) \text { of } \\
\text { patients with CRE } \\
(n=305)\end{array}$ \\
\hline \multicolumn{2}{|l|}{ Organism } \\
\hline Klebsiella pneumoniae & $277(91)$ \\
\hline Escherichia coli & $19(6)$ \\
\hline Klebsiella oxytoca & $5(2)$ \\
\hline Enterobacter cloacae & $3(1)$ \\
\hline Proteus mirabilis & $1(<1)$ \\
\hline \multicolumn{2}{|l|}{ Infection } \\
\hline Overall & $180(59)$ \\
\hline Urinary tract infection & $101(33)$ \\
\hline Pneumonia & $23(7)$ \\
\hline Bloodstream infection & $21(7)$ \\
\hline Decubitus ulcer & $15(5)$ \\
\hline Abscess & $5(2)$ \\
\hline Osteomyelitis & $1(<1)$ \\
\hline Other/unknown & $14(5)$ \\
\hline \multicolumn{2}{|l|}{ Colonization } \\
\hline Overall & $125(41)$ \\
\hline Urine & $81(27)$ \\
\hline Sputum & $28(9)$ \\
\hline Perirectal & $7(2)$ \\
\hline Other/unknown & $9(3)$ \\
\hline \multicolumn{2}{|l|}{ Location of acquisition } \\
\hline Community onset, healthcare associated & $184(60)$ \\
\hline Hospital onset, healthcare associated & $104(34)$ \\
\hline Community acquired & $17(6)$ \\
\hline
\end{tabular}




\section{TABLE 2}

Survey of Microbiology Laboratory Practices from the Duke Infection Control Outreach Network, 2008-2012

\begin{tabular}{lc}
\hline Variable & $\begin{array}{c}\text { No. }(\boldsymbol{\%}) \\
\text { of hospitals } \\
(\boldsymbol{n}=\mathbf{2 5})\end{array}$ \\
\hline Adoption of 2010 CLSI breakpoints & \\
Yes & $5(20)$ \\
No & $20(80)$ \\
Automated MIC system & \\
Vitek 2 & $13(52)$ \\
Microscan & $12(48)$ \\
Verification of carbapenemase & \\
Modified Hodge test & $10(40)$ \\
Forward to reference laboratory & $13(52)$ \\
No verification performed & $2(8)$ \\
Report CRE to infection control & \\
Yes & $23(92)$ \\
No & $2(8)$ \\
Active CRE surveillance & \\
Yes & $0(0)$ \\
No & $12(100)$ \\
\hline
\end{tabular}

NOTE. CLSI, Clinical and Laboratory Standards Institute; CRE, carbapenem-resistant Enterobacteriaceae; MIC, minimum inhibitory concentration. 


\section{TABLE 3}

Variability in Carbapenem-Resistant Enterobacteriaceae (CRE) Detection as a Function of Microbiology Laboratory Practices

\begin{tabular}{lrcc}
\hline Hospitals & $\begin{array}{c}\text { Mean }( \pm \text { SD) CRE } \\
\text { No. of } \\
\text { hospitals }\end{array}$ & $\begin{array}{c}\text { Cetected per 100,000 } \\
\text { patient-days per hospital }\end{array}$ & $\boldsymbol{P}^{\boldsymbol{a}}$ \\
\hline Reporting CRE to infection control & 22 & $1.64 \pm 2.30$ & .16 \\
Not reporting CRE to infection control & 2 & 0 & .01 \\
Using Vitek 2 system & 12 & $2.47 \pm 2.78$ & .07 \\
Using Microscan system & 12 & $0.54 \pm 0.85$ & \\
Performing a modified Hodge test & 9 & $2.36 \pm 3.34$ & .01 \\
Sending strains to outside laboratory & 13 & $1.00 \pm 1.06$ & \\
Adopting 2010 CLSI breakpoints & 5 & $3.62 \pm 3.52$ & \\
Not adopting 2010 CLSI breakpoints & 19 & $1.08 \pm 1.61$ & \\
\hline
\end{tabular}

NOTE. CRE is endemic in one of the study hospitals, which was therefore excluded from this analysis. CLSI, Clinical Laboratory and Standards Institute; SD, standard deviation.

${ }^{a}$ Calculated using Student $t$ test. 